Análisis comparativo de la utilización de refrigerantes alternativos en un chiller

\title{
Análisis comparativo de la utilización de refrigerantes alternativos en un chiller
}

\section{Comparative analysis of the use of alternative refrigerants in a cooler}

\section{Análise comparativa do uso de refrigerantes alternativos em um resfriador}

Telmo J. Moreno-Romero ${ }^{\mathrm{I}}$

telmomoreno@hotmail.com

Ana C. Cadena-Nogales II

ana.cadena@espoch.edu.ec

John G. Vera-Luzuriaga III

john.vera@espoch.edu.ec

Lenin S. Orozco-Cantos IV

1sorozco@espoch.edu.ec

Recibido: 25 de agosto de 2017 * Corregido: 29 de octubre de $2017 *$ Aceptado: 10 de diciembre de 2017

ı. Master en Ciencias, Especialista en Computación Aplicada al Ejercicio Docente, Ingeniero Mecánico, Docente de la Escuela Politécnica de Chimborazo, Riobamba, Chimborazo, Ecuador.

II. Master of Science in Chemical Engineering, Ingeniera Química, Docente de la Escuela Politécnica de Chimborazo, Riobamba, Chimborazo, Ecuador.

III. Master Universitario en Ingeniería Mecatrónica, Ingeniero en Electrónica, Control y Redes Industriales, Docente de la Escuela Politécnica de Chimborazo, Riobamba, Chimborazo, Ecuador.

ıv. Magister en Diseño Producción y Automatización Industrial, Ingeniero Mecánico, Docente de la Escuela Politécnica de Chimborazo, Riobamba, Chimborazo, Ecuador. 


\title{
Resumen
}

Los sistemas de refrigeración representan un gran consumidor de energía por lo cual en la búsqueda de eficiencia en el consumo energético no solo se debe estudiar el diseño de nuevos sistemas sino también se deben analizar y mejorar los sistemas existentes. En este caso se analiza la utilización de distintos refrigerantes secundarios en equipos chiller. Este análisis se centra en el estudio del comportamiento y características de distintos refrigerantes secundarios con la finalidad de mostrar alternativas de refrigerantes secundarios al momento de seleccionar un fluido para una aplicación, dependiendo de los requerimientos del usuario.

Palabras clave: Chiller; anticongelante; refrigerante secundario; refrigeración; sistemas indirectos; nano fluidos.

\begin{abstract}
Refrigeration systems represent a large consumer of energy, so in the search for efficiency in energy consumption not only should the design of new systems be studied, but also the existing systems should be analyzed and improved, as in this case it is analyzed the use of different secondary refrigerants in chiller equipment, this analysis focuses on the study of the behavior and characteristics of different secondary refrigerants in order to show alternatives of secondary refrigerants when selecting a fluid for an application depending on the user's requirements.
\end{abstract}

Keywords: Chiller; antifreeze; secondary refrigerant; refrigeration; indirect systems; nano fluids.

\section{Resumo}

Os sistemas de refrigeração representam um grande consumidor de energia, portanto, na busca da eficiência energética, não somente o projeto de novos sistemas deve ser estudado, mas também os sistemas existentes devem ser analisados e aprimorados. Neste caso, o uso de diferentes refrigerantes secundários em equipamentos de refrigeração é analisado. Esta análise centra-se no estudo do comportamento e das características dos diferentes refrigerantes secundários, a fim de mostrar alternativas de refrigerante secundário ao selecionar um fluido para uma aplicação, de acordo com os requisitos do usuário.

Palavras chave: Chiller; anticongelante; refrigerante secundário; refrigeração; sistemas indiretos; Nano fluidos. 


\section{Introducción}

El alto consumo de los sistemas de refrigeración hace necesario que se estudien los mismos con la finalidad de mejorarlos, de esta manera es necesario que se tomen en cuenta aspectos tanto térmicos como económicos. Este sistema funciona mediante la compresión de vapor (Franco Lijó, 2006) y dentro de los componentes que realizan un proceso térmico de este sistema se encuentran el compresor, condensador, válvula de expansión y el evaporador. Todos esos componentes son indispensables, ya que de no existir un elemento no se puede cerrar el ciclo térmico y no se realizaría la función de refrigeración.

Para un estudio adecuado de refrigeración también se hace factible la revisión de los sistemas y refrigerantes en aspectos físico-químicos, biológicos y microbiológicos, principalmente cuando uno de los fines de la refrigeración es la conservación de sustancias (Barreiro, y otros, 2006) (Hernández, 2010).

Cuando el sistema de refrigeración se orienta al uso HVAC (Heating, Ventilating and Air Conditioning), el estándar ASHRAE-90.1 intenta ser estrictamente neutral y recomienda ciertos tipos de sistemas desde el punto de vista de la racionalización y eficiencia energética.

La norma china también tiende a dar más orientación de diseño, en lugar de simplemente enumerar los requisitos de la norma. (Hong, 2009). Para las calderas, el estándar tiene un requisito de eficiencia fijo independiente del tamaño de la caldera. La Norma requiere que las calderas que funcionan con combustible / gas tengan una eficiencia del 89\%. Para enfriadores (chillers) tiene requisitos más altos (en un 8-18\%) de COP (coeficiente de eficiencia energética en modo calefacción) e IPLV. (Hong, 2009) (ASHRAE, 2015)

Otra forma de utilización de los equipos chiller es la tecnología de refrigeración por adsorción está atrayendo cada vez más atención en los últimos años porque puede ahorrar energía y es amigable con el medio ambiente. Los ciclos de adsorción pueden ser impulsados por calor residual de baja calidad o energía solar por debajo de $80^{\circ} \mathrm{C}$. No tienen que usar clorofluorocarbonos (CFC) que agotan la capa de ozono y no necesitan electricidad o combustibles fósiles como fuentes de manejo. (Chang, y otros, 2009) (Chang, y otros, 2007) 
Análisis comparativo de la utilización de refrigerantes alternativos en un chiller

El equipo de refrigeración chiller funciona como un enfriador indirecto que es utilizado en el campo comercial, en la industria de alimentos, en la industria en general como enfriador de equipo y aplicaciones en aire acondicionado. El chiller al ser un equipo de refrigeración indirecto el refrigerante primario o fluido de trabajo no entra en contacto con el medio refrigerado y este sistema de funcionamiento tiene la ventaja de disminuir fugas y disminuir la carga del refrigerante primario, por lo cual es utilizado para enfriar medios remotos sin que se eleve el costo de la instalación (Melinder, 2010), (Saha, y otros, 1997)

El fluido de trabajo enfría a un fluido secundario conocido como medio de enfriamiento o refrigerante secundario, este fluido es impulsado mediante una bomba hacia el proceso mismo que se lleva a cabo en la planta. Durante el intervalo de enfriamiento del proceso de la planta industrial, el refrigerante secundario no cambia de fase, por lo que al realizar la selección de un anticongelante para formar un refrigerante secundario se debe tomar en cuenta que posea propiedades favorables para producir una transferencia eficiente de calor (Prabhakaran, y otros, 2016), (Powell, y otros, 2013)

En la actualidad existe gran cantidad de refrigerantes secundarios, pudiendo separarlos en refrigerantes de origen orgánico, y refrigerantes de origen inorgánico. Hoy en día también es posible encontrar refrigerantes con nanopartículas suspendidas en un fluido base, a esta mezcla de las dos sustancias se le conoce como nanofluidos, en el cual mediante la adición de nanopartículas al fluido base se obtiene cambios en las propiedades de transporte y el las características de transferencia de calor (Prabhakaran, y otros, 2016), (Wang, y otros, 2007), (Liu, y otros, 2009)

Un fluido secundario debe cumplir con ciertos requerimiento o parámetros para ser considerado como tal, el fluido debe poseer fácil y segura manipulación, costo, toxicidad, corrosión, inflamabilidad, contaminación (Melinder, 2000) y que sea factible su implementación en los sistemas existes.

Estudios muestran que en etilenglicol con suspensiones $0.01(\% \mathrm{~V})$ de "multiwalled carbón nanotubes". La conductividad térmica mejora en un $12.4 \%$. En agua en suspensiones $(1.5 \% \mathrm{~V})$ de "multiwalled carbón nanotubes" la conductividad térmica mejora en un 17.8 \%. (Liu, y otros, 2009).

\section{Metodología}

El proceso de refrigeración es un proceso que está influenciado por varios parámetros. Los mismos deben ser analizados con la finalidad de contar con sistemas eficientes de alto valor de COP- Para esto 
se debe examinar el comportamiento del sistema y adaptarlo a las condiciones de trabajos reales con la finalidad de que los resultados puedan ser aplicados directamente en aplicaciones industriales, buscar mejoras en el rendimiento de cada elemento del sistema, analizar el comportamiento de los refrigerantes considerando que estos sean seguros para el medio ambiente, detectar averías y degradaciones que afectan al sistema (Bejarano, y otros, 2013) y analizar las condiciones del medio circundante y del fluido a refrigerar tomando en cuenta la temperatura a la que estos se encuentran. (Ruz, y otros, 2016) (Díaz Torres, 2014) (Yu, 2017)

Entre los distintos sistemas de refrigeración, el más utilizado es el sistema de refrigeración por compresión de vapor (Franco Lijó, 2006) y entre los principales procesos que se lleva a cabo en este proceso se encuentran el proceso de compresión, condensación, expansión y evaporación. Este sistema es el que utiliza el equipo chiller para llevar a cabo el proceso de refrigeración.

El equipo chiller es un equipo de refrigeración industrial, que trabaja como un sistema de refrigeración indirecto, ya que el refrigerante no está en contacto directo con el proceso, por lo que enfría a otro fluido que está en contacto con el proceso, este fluido generalmente es agua de ahí que se conoce al chiller como planta enfriadora de agua.

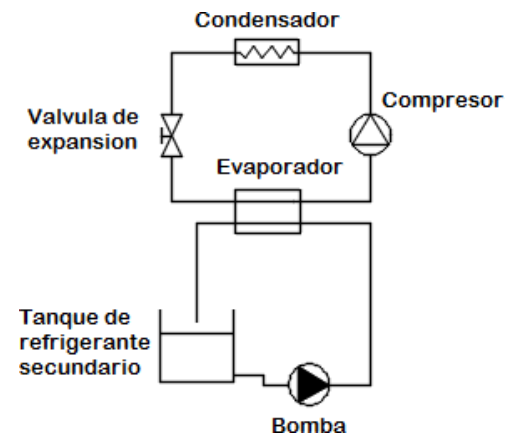

Figura 1: Esquema del equipo chiller. Realizado por: Autores

En la figura 1, se puede observar la disposición general de los elementos de un chiller los mismos que cumplen una función específica en el ciclo de refrigeración y en la transferencia de calor entre el fluido primario y el refrigerante secundario. (Thangavelu, 2017)

Dependiendo de las necesidades se puede reemplazar el agua por algún otro tipo de refrigerante. El mismo deberá ser compatible con los materiales que están en contacto con el refrigerante secundario. 
Los materiales de un chiller equipado con un sistema de adquisición de datos, que se encuentran en contacto con el refrigerante secundario, son los que se muestran en la Tabla 1 con su respectivo material. Para el análisis de compatibilidad del fluido con el material se debe analizar principalmente el nivel de corrosividad de la solución acuosa para el material determinando así su compatibilidad.

Tabla 1: Materiales del equipo chiller que se encuentran en contacto con el refrigerante secundario.

\begin{tabular}{|l|l|}
\hline Elemento & Material \\
\hline Válvula de pie & Bronce \\
\hline Canastilla de válvula. & Acero Inoxidable \\
\hline Tubería de conducción & Polipropileno \\
\hline Accesorios del sistema de conducción & Polipropileno \\
\hline Intercambiador de calor & Acero Inoxidable \\
\hline Paletas del caudalímetro & Polifluoruro de vinilideno \\
\hline Rotor del sensor caudalímetro & Titanio \\
\hline Rodete de bomba & Polipropileno \\
\hline Tanque de almacenamiento & Acero Inoxidable \\
\hline
\end{tabular}

\section{Realizado por: Autores}

En el caso específico de los elementos del chiller que trabajan con el fluido secundario se encuentra el evaporador que es donde se lleva a cabo la transferencia de calor entre el refrigerante primario y refrigerante secundario y la bomba que es la encargada de impulsar el fluido hacia el proceso que se lleva a cabo en la planta industrial.

\section{Evaporador de placas}

Es un intercambiador de calor que absorbe calor latente de un medio de enfriamiento. Para el caso de sistemas de enfriamiento como el chiller dicho fluido puede ser agua o algún tipo de salmuera y en el que generalmente se utiliza es evaporadores de placas.

Está formado por varias placas dispuestas en un bastidor, las placas cuentan con pliegues en su superficie haciéndola más rígida y direccionando el flujo, para evaporadores de gran capacidad las placas se encuentran soldadas por pares y se sujetan al bastidor mediante pernos.

Debido a su facilidad de desmontaje este tipo de evaporadores son muy utilizados en sistemas de refrigeración de plantas industriales alimenticias ya que se puede llevar un gran control de la higiene de las placas y evitar contaminación de los alimentos. 


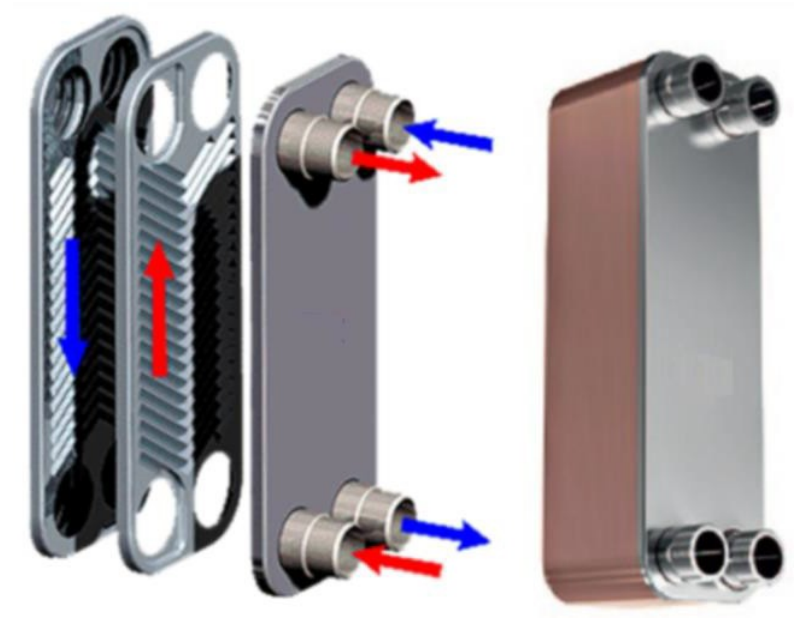

Figura 2: Evaporador de placas. Fuente:

(https://www.tiendafriguscenter.com/detalles_Intercambiadores-de-Calor)

Como se muestra en la figura 2 el refrigerante circula entre dos placas soldadas y el medio de enfriamiento circula por el espacio entre dos pares de placas, realizándose la trasferencia de calor entre el refrigerante y el medio de enfriamiento.

Las características de los fluidos, la pared del evaporador y adicionalmente la diferencia de temperatura son factores que determina la cantidad de calor transferido como se muestra en la ecuación siguiente según

$$
Q=A \cdot U \cdot \Delta T
$$

Dónde:

Q: Calor transferido [W]

A: Área de transferencia de calor $\left[\mathrm{m}^{\wedge} 2\right]$

$\mathrm{U}$ : Coeficiente global de transferencia de calor $\left[\mathrm{W} /{ }^{\circ} \mathrm{C}-\mathrm{m}^{\wedge} 2\right]$

$\Delta \mathrm{T}$ : Diferencia de temperatura entre refrigerante y medio condensante $\left[{ }^{\circ} \mathrm{C}\right]$

Las propiedades del fluido de enfriamiento influyen sobre el coeficiente global de transferencia de calor y este a su vez en la transferencia de calor. (Bahrami, 2017) (Yu, 2018) 
Se sabe que la COP del enfriador no se mide directamente, pero se calcula a partir de las variables dependientes medidas. El coeficiente de rendimiento del enfriador, COP, es una relación entre la capacidad de refrigeración del enfriador $(\mathrm{Q})$ y el consumo de energía del enfriador $(\mathrm{P})$, representada de la siguiente manera:

$$
C O P=\frac{Q}{P}
$$

Donde $\mathrm{P}$ es el consumo de energía del enfriador $(\mathrm{kW})$, que consiste en compresor, potencia de los ventiladores $\left(P_{F}\right)$, potencia del condensador $\left(P_{C}\right)$ y potencia de la bomba $\left(P_{P}\right)$, está dado por:

$$
P=P_{C}+P_{F}+P_{P}=\sqrt{3 V} I_{C} \cos \theta+\sqrt{3 V} I_{F} \cos \theta+\sqrt{3 V} I_{P} \cos \theta
$$

Donde el factor de potencia debe tender a los 0.8. (Kabeel, 2017)

La bomba de impulsión del refrigerante secundario es el componente encargado de impulsar y mantener la recirculación continua del medio de enfriamiento en este caso de la salmuera hacia el proceso, para un adecuado funcionamiento del equipo chiller se debe contar con una apropiada potencia de bombeo ya que a medida que el refrigerante se enfrié en este se produce un incremento de la viscosidad por lo que se requerirá mayor potencia para impulsar el fluido. (Sam, y otros, 2017) La potencia requerida para bombear un medio de enfriamiento como agua o cualquier salmuera está dada por la siguiente ecuación.

$$
P=\frac{Q \times H_{\operatorname{man}} x \gamma}{1000 \times \eta}
$$

Dónde:

P: Potencia de bombeo [W]

Q: Caudal [l/s] 
$\mathrm{H}_{\text {man: Altura manometrica }[\mathrm{m}]}$

$\gamma$ : Peso especifico de la salmuera $\left[\mathrm{N} / \mathrm{m}^{3}\right]$

$\eta$ : Rendimiento $[-]$

La altura manométrica es una medida que resulta de sumar la altura de cabeza de bombeo, las longitudes equivalentes de cada accesorio y las perdidas por rozamiento en tubería del sistema de bombeo. Para el cálculo de las perdidas por rozamiento es necesario utilizar la ecuación de Darcy Weisback:

$$
H_{r p}=f \frac{L}{D} \frac{v^{2}}{2 g}
$$

Dónde:

$\mathrm{H}_{\mathrm{rp}}$ : Perdida por rozamiento [m]

f: Coeficiente de fricción [-]

L: Longitud de tuberia [m]

D: Diametro de tuberia $[\mathrm{m}]$

v: Velocidad del fluido [m/s]

g: Gravedad $\left[\mathrm{m} / \mathrm{s}^{2}\right]$

El coeficiente de fricciona se puede obtener del diagrama de Moody o de ecuaciones empíricas como la ecuación de Fanno, el coeficiente de rozamiento está en función del número de Reynolds y de la rugosidad relativa, a su vez el número de Reynolds depende de la viscosidad del fluido.

Debido a características de los fluidos en estado líquido se conoce que la viscosidad de las salmueras aumenta al disminuir la temperatura por lo tanto al incrementarse la viscosidad análogamente aumenta 
la perdida por rozamiento haciendo que a medida que se enfríe la salmuera se necesita mayor potencia de bombeo.

\section{Desarrollo}

Para el análisis de los posibles resultados hay que tomar en cuenta aspectos fundamentales del empleo de cada solución acuosa usada como medio de enfriamiento secundario en el ciclo de refrigeración pues a lo largo de los años investigaciones han demostrado ciertos parámetros tales como el cuidado al generar un impacto ambiental debido al índice de toxicidad, la corrosión que pueda ocasionar a las tuberías y la energía necesaria para el bombeo ya que al usar un sistema de refrigeración no directa si no por medio de un refrigerante secundario, donde las instalaciones que realizan el ciclo frigorífico o de enfriamiento necesitan de una bomba conectada al evaporador para mover al refrigerante secundario, por lo cual es de suma importancia la capacidad de bombeo (Alé, 2000), y el consumo de energía para el bombeo del respectivo fluido, como se muestra en la tabla 2 en el cual se muestra el valor de energía relativa de varios fluidos que se pueden usar como refrigerante secundario.

Tabla 2: Refrigerantes secundarios y factor de energía de bombeo.

\begin{tabular}{|l|l|}
\hline $\begin{array}{l}\text { Refrigerante } \\
\text { secundario }\end{array}$ & $\begin{array}{l}\text { Factor } \\
\text { energía }\end{array}$ \\
\hline Agua-Amoniaco & 1 \\
\hline Metanol & 1,078 \\
\hline Propilenglicol & 1,142 \\
\hline Etilenglicol & 1,25 \\
\hline Cloruro de sodio & 1,295 \\
\hline Cloruro de calcio & 1,447 \\
\hline d-Limoneno & 2,406 \\
\hline Cloruro de Metileno & 3,735 \\
\hline Tricloroetileno & 4,787 \\
\hline R-11 & 5,022 \\
\hline
\end{tabular}

Fuente: ASHRAE, Handbook - Refrigeration, 2010 (ASHRAE, 2010)

Desde el punto de vista térmico los fluidos considerados como refrigerantes secundarios deben poseer buenas propiedades térmicas, lo que significa un correcto valor de calor específico, viscosidad, temperatura de congelación, presión de vapor entre las más importantes

Las alternativas de posibles fluidos usados como refrigerantes secundarios y sus resultados están: 
Análisis comparativo de la utilización de refrigerantes alternativos en un chiller

Soluciones de agua y propilenglicol: Es un fluido soluble en agua que es considerado seguro para usar en alimentos, medicamentos y cosméticos. Sus atractivas propiedades como solvente, humectante, anticongelante combinado con su relativo bajo nivel de toxicidad, han dirigido su extensa utilización en un largo número de productos industriales y comerciales. La FDA clasifica al propilenglicol como un aditivo que es generalmente reconocido como seguro para su uso en alimentos. Propilenglicol es comúnmente el mejor constituyente de anticongelantes y líquidos de deshielo de carros, aviones y barcos (Wexler, 2014). Es una mezcla que se comporta bien cuando se la usa hasta temperaturas de $15^{\circ} \mathrm{C}$ pero tiene viscosidad alta a bajas temperaturas lo cual dificulta el bombeo aumentando potencia en la bomba y disminuyendo caudal.

Soluciones de agua y etilenglicol: Es menos viscoso y se comporta estable hasta temperaturas de $15^{\circ} \mathrm{C}$, genera un riesgo ambiental debido a su alta toxicidad con la polución ambiental y debido es a sus características de no color y no olor representa un alto riesgo para el operario de estos sistemas.

Soluciones de agua con alcohol etílico y metílico: Su punto de ebullición es bajo, generan riesgo por su inflamabilidad, riesgo ambiental nivel de toxicidad, por lo que su uso se encuentra restringido a industrias donde existen riesgos similares y que las mismas cuenten con sistemas que permitan contrarrestar los riesgos de explosión.

Soluciones de cloruro de calcio y cloruro de sodio: Tienen muy buena capacidad de transferencia de calor y tiene gran aplicabilidad a nivel industrial, su temperatura de congelación es de aproximadamente $-55^{\circ} \mathrm{C}$, esta salmuera no es tóxica pero no se recomienda su contacto directo con alimentos que no son herméticamente cerrado a diferencia de la salmuera de cloruro de sodio, este anticongelante resulta ser no compatible para aplicaciones con acero inoxidable ya que es posible que se produzca corrosión (Ignatowicz, 2008), lo que generan altos daños en la integridad de las instalaciones.

Cloruro de sodio: Posee buenas propiedades termodinámicas y baja toxicidad por lo que puede estar en contacto con alimentos, con la desventaja de ser altamente corrosivo y que su punto de congelación es relativamente elevado en comparación con otros refrigerantes secundarios, siendo esta temperatura aproximadamente de $-20^{\circ} \mathrm{C}$ (Carrier Air Conditioning Company, 1980). 
Análisis comparativo de la utilización de refrigerantes alternativos en un chiller

Soluciones no acuosas: Entre las soluciones no acuosas comúnmente usados como refrigerante secundario se encuentran los hidrocarburos y halocarburos fluorados y clorados los mismos que son de costo elevado y se utilizan en servicios de baja temperatura inferior a $-40^{\circ} \mathrm{C}$ (Carrier Air Conditioning Company, 1980), lo que significa que el fluido tiene relativamente bajas temperaturas de congelamiento

Nanofluidos: Estos presentas varias aplicaciones en cuanto a lo referente a refrigeración son la composición de la solución de agua y una salmuera más la adición de nanopartículas.

Se desarrolla esta investigación con el fin de aumentar la transferencia de calor del fluido en sistemas de refrigeración ya que se sabe que los sólidos son mejores en cuanto a conductividad térmica en comparación a los líquidos pero generalmente generan mucha sedimentación por la suspensión de las partículas en el agua, generando taponamientos en los conductos y aumentando la demanda de bombeo es decir aumenta la potencia de la bomba, por lo cual se optó por añadir partículas de sólidos nanométricas, de esta manera pueden permanecer en suspensión mayor tiempo sin generar sedimentación gracias al movimiento Browniano de los fluidos, hasta el momento es un campo no tan explorado se sabe que se han hecho pruebas con algunos materiales como: Oxido de cobre, Alúmina, Óxido de titanio, Óxido de hierro, Oro, Plata, Silicio, Grafeno.

Estas han mostrado una cuantificación mayor en lo referente a conductividad y convección en la transferencia de calor mejorando y superando los análisis teóricos en cuanto a la experimentación ya que solo se ha experimentado con pocos de ellos los estudios están solo en papel la mayoría de estos aún no han sido experimentados en lo referente a la industria. (Martinez-Pérez, 2014)

Una de las aplicaciones es el estudio de las nanopartículas, es en el uso en el sistema de refrigeración de un vehículo para tener mayor eficiencia al permitir funcionar a mayor temperatura y obtener mayor potencia sin dejar de cumplir con las normas de emisión. La alta conductividad térmica en radiadores puede permitir la reducción y ahorro de combustible al disminuir desgaste de bombas y compresores.

Desde el punto de vista térmico el propilenglicol es el anticongelante que relativamente tiene menor coeficiente de transferencia de calor, debido a que la transferencia de calor está influenciada con varios parámetros que deben ser analizados para determinar su verdadero comportamiento es necesario 
Análisis comparativo de la utilización de refrigerantes alternativos en un chiller

experimentar con los fluidos con la finalidad de obtener su comportamiento real en el sistema y determinar tiempos de enfriamiento y los costos producidos por el consumo de uno u otro refrigerante.

\section{Conclusiones}

Una característica fundamental de los refrigerantes secundarios es su punto de congelamiento hasta el cual la transferencia de calor se da por un enfriamiento sensible al no existir un cambio de fase, la selección del refrigerante secundario depende de la aplicación en la que vaya a trabajar el refrigerante y la mejora del proceso de intercambio de calor, salvaguardando la integridad física tanto de los equipos como del recurso humano a cargo de la operación.

En la búsqueda de conseguir equipos más eficientes de refrigeración hay que tomar en cuenta criterios económicos y criterios ambientales, se necesita que se realice un análisis más profundo del comportamiento que tienen los distintos anticongelantes mediante ensayos, el propilenglicol debido a sus características de no contaminación, no toxicidad y relativo bajo coeficiente de energía para ser bombeado hace que se realice las pruebas en el equipo chiller con la finalidad de determinar su comportamiento real en el equipo en condiciones de trabajo.

En lo referente a nanofluidos son la nueva era para generar un desarrollo y una innovación en la transferencia de calor ya que al aumentar en la solución nanopartículas estas no generan sedimentación permitiendo que el equipo trabaje a condiciones normales sin sobre-esfuerzos y haciendo que la conductividad y la convección se aumenten en un rango considerable haciendo más eficiente a la solución. Cabe resaltar que estos estudios están aún en proceso generando un campo que aún no ha sido explorado en su totalidad dando la apertura de realizar futuros estudios referentes a los nanofluidos su aplicación y resultados para poder usarlos en la industria.

Una determinación real del comportamiento de un fluido secundario es posible de obtener mediante una experimentación con el fluido, en la que el ensayo muestra datos de temperatura, caudal, y tiempos lo que mediante una extrapolación de datos se puede graficar las curvas de enfriamiento para el refrigerante lo que ayuda interpretar el comportamiento del refrigerante en el equipo y su respectiva comparación con otro fluido con la finalidad de conseguir un sistema eficiente de refrigeración. 


\section{Referencias bibliográficas}

Alé, J.V.,\& Santos,G.A. 2000. Congresso de Ar Condicionado Refrigeracao, Aquecimento e Ventilacao do Mercosul. 2000.

ASHRAE. 2015. ASHRAE, Advance energy desing guides. [En línea] ASHRAE, 2015. www.ashrae.org/aedg..

- 2010. Handbook - Refrigeration. Atlanta: American Society of Heating, Refrigerating and AirConditioning Engineers, Inc., 2010. págs. 46-66. 978-1-933742-82-3.

Bahrami, M. 2017. Evaluation of low - pressure flooded evaporator performance for absorption chillers. 2017. págs. 144-158. Vol. 122.

Barreiro, Jose y Sandoval, Aleida. 2006. Operaciones de conservacion de alimentos por bajas temeraturas. Caracas: Editorial Equinoccio, 2006.

Bejarano, Guillermo, y otros. 2013. Modelado simplificado y orientado al control de sistemas de redrigeración. Terrassa: s.n., 2013.

Carrier Air Conditioning Company. 1980. Manual de Aire Acondicionado. Barcelona: Marcombo, 1980. págs. 389-392.

Chang, C, Whang, C y Shieh, C. 2007. Experimental study of a solid adsorption cooling system using flat-tube heat exchangers as adsorption bed. 2007. págs. 2195-2199. Vol. 27.

Chang, W, Wang, C y Shied, C. 2009. Desing and performance of a solar powered heating and cooling system using silica gel adsorption chiller. 2009. págs. 2100-2105. Vol. 29.

Diaz Torres, Y. 2014. Analisis energetico de un sistema hibrido de produccion de frio. 2014. págs. 38-49. Vol. XXXVI.

Franco Lijó, Juan Manuel. 2006. Manual de Refrigeración. Barcelona: Reverte, 2006. págs. 1-185.

—. 2006. Manual de Refrigeración. Barcelona: Reverte, 2006. 
Análisis comparativo de la utilización de refrigerantes alternativos en un chiller

Hernández, Eduardo. 2010. Fundamentos de aire acondicionado y refrigeración. Mexico: LIMUSA, 2010 .

Hong, Tianzhen. 2009. A close look at the China Design Standard for Energy Efficiency of Public Buildings. 2009, Vol. 41, 41, págs. 426-435.

Ignatowicz, Monika. 2008. Corrosion aspects in indirect systems with secondary refrigerants. Master of Science Thesis. Stockholm, Sweden: s.n., 2008. pág. 69.

Kabeel, A.E. 2017. Performance evaluation of energy efficient evaporatively ais-cooled chiller. 2017. págs. 204-213. Vol. 22.

Liu, M. S, Lin, M y Liaw, J. 2009. Performance Augmentation of a Water Chiller System Using Nanofluids. ASHRAE Transactions. 2009, Vol. 115, págs. 581-586.

Martinez-Pérez, F.J. 2014. III Seminario Internacional de Ciencias Aplicadas. 2014. págs. 4-6.

Melinder, Å. 2010. Properties and Other Aspects of Aqueous Solutions Used for Single Phase and Ice Slurry Applications. s.1. : Elsevier, 2010. págs. 1506-1512. Vol. 33.

—. 2000. Update on Secondary Refrigerants for Indirect Systems. 2000, Vol. 2, págs. 1-2.

Powell, Kody, y otros. 2013. Optimal chiller loading in a district cooling system with thermal energy storage. 2013. págs. 445-463. Vol. 50.

Prabhakaran, M, y otros. 2016. Development and Assessment of Ceria-Propylene Glycol Nanofluid as an Alternative to Propylene Glycol for Cooling Applications. s.1. : Elsevier, 2016. págs. 329-335. Vol. 102.

Ruz, Mario L, y otros. 2016. Planta experimental para supervisión y control del ciclo de refrigeración por compresion de vapor. Madrid: CEA-IFAC, 2016. págs. 308-315.

s/a. 2012. Cost-benefit analysis of using cold thermal energy storage systems in building applications. 2012, Vol. 14, 14, págs. 493-501.

Saha, B y Kashiwagi, T. 1997. Experimental investigation of an advanced adsorption refrigeration. 1997. págs. 50-58. 
Sam, K, Lam, K y Tham, C. 2017. Distributed Pumping Chilled Water Hydronic System for Airconditioning Systems. 2017. págs. 88-93. Vol. 143.

Stoecker, Wilbert F. 1998. Industrial Refrigeration Handbook. New York: McGraw-Hill, 1998. págs. $113,169-173,248,653-664$.

Thangavelu, Raj. 2017. Energy optimization methodology of multichiller plant in commercial buildings. 2017. págs. 64-76. Vol. 123.

Wang, Shan K. 2000. Handbook of Air Conditional and Refrigeration. Second. New York: McGrawHill, 2000. págs. 403-462.

Wang, X. Q y Mujumdar, A.S. 2007. Heat transfer characteristics of nanofluids: a review. 2007, Vol. 46, 1, págs. 1-19.

Wexler, Philip. 2014. Encyclopedia Of Toxicology. London: Elsevier, 2014. págs. 3766-3767, 1915. Yu, W. F. 2017. Review of standars of energy performance of chhiller systems serving commercial buildings. 2017. págs. 144-158. Vol. 122.

—. 2018. Theorical and experimental analyses of mist precooling for an air-cooled chiller. 2018. págs. 112-119. Vol. 130. 